\title{
Panel Discussion II
}

\author{
Panel: F. Allard, A. Batten, E. Budding, E. Devinney, P. Eggleton, A. Hatzes, \\ I. Hubeny, W. Kley, H. Lammer, A. Linnell, V. Trimble, and R. E. Wilson
}

\section{Discussion}

I. Hubeny: Today, the discussion will be open to the general audience. In Sessions C, D, and $\mathrm{E}$, we have talked about models and modelling techniques so I expect the discussion will focus on these topics.

V. TRimble: Comparing codes is enormously important - we learned that decades ago with stellar structure and evolution. And it's important that groups comparing codes be sure that they have the same physics before they decide whether they are agreeing or disagreeing. If you have different physics in your models, of course you get different answers. That requires possibly more openness in the early stages of developing codes than is usual. The separate codes need to be compared with each other, with the observations, and with the tables. If you cannot reproduce earlier analytic results, something is wrong.

A. Hatzes: For someone who doesn't do any light curve modelling you said, "Compare the codes." Are there standard tests? I mean they should all be compared to the exact same thing.

H. LAMMER: I agree with this completely because this was done in the case of planetary atmosphere interaction studies, at least for the Solar system. Several teams got together to apply hybrid codes for studying the loss of Mars' atmosphere. You also need people who write the codes to discuss their codes with each other, and you need also the exact similar input, because if you don't know what the input is: let's say if you've got 10 codes, you will get 10 different results. I don't know how difficult it will be. You can never find a detailed description of the codes in any paper.

A. Burrows: At Los Alamos, for example - Livermore, they have standard verification and validation protocols for various types of codes. It's easiest in spherical symmetry, it's easiest in the planar case. There are hydro-calculations you can do in $1 \mathrm{D}$ and $2 \mathrm{D}$, in particular, that are almost analytic; some are analytic and some are almost analytic, and have been tested for a long time. You can do resolution studies, but there is a cultural problem as well, that Virginia alluded to, that people don't want to really share their errors or the fact that they have them. So over time, people in the field of numerical simulation, particularly hydro, but also radiative transfer to some degree, have reached a comfort level where this is done much more, and it's also expected much more. So, the number of people doing these calculations has gone up significantly in the last 30 years. In the 1980s for example, people weren't that confident, and there was very little collaboration and communication and verification and validation.

S. Mochnacki: When we first did these codes, we used the tables that were developed for spherical stars, and I also worked on doing the reflection effect, the geometrical aspects of it. And it turned out there was a fellow named Napier who worked it out analytically 
for spherical stars, and got it right! Many people like Kopal had never gotten it right before because they got lost in the analysis and made too many algebraical errors. So, we do stand on the shoulders of giants, and that early work is vital to test the things we write ourselves.

I. Hubeny: Obviously, different codes need different comparisons. I have experience with testing and comparing stellar atmosphere codes. Once we organized a session at a conference in Trieste, we assigned reasonably the same model, meaning the same parameters. People were asked to compute models, which didn't help at all, because the models were very similar, but there were differences impossible to resolve at the conference because of the vast amount of data you need from an atmosphere. So, I did an independent comparison with Klaus Werner and Stefan Dreizler in Tuebingen. I invited them for three weeks to Goddard (when I was working there), and it took three weeks to work out various small things, and after three weeks we finally ended up having a very reasonable agreement. We then did the same thing with John Hiller with CMFGEN that worked for the static approximation. Again, it took us a lot of time, a lot of effort, to tweak all those too. It's an extremely valuable experience, not only an exercise, not only for comparison, but you learn a lot about your mistakes and the other party learns about their mistakes and you get much more confidence in the codes. So, I highly recommend it. Now, for different codes we will need different sorts of comparisons, but it's an extremely important and valuable exercise to do. We also did a very detailed comparison with Detlev Koester and Pierre Bergeron on white dwarf models, because people use them for calibrations. It was again a huge work and we agreed to publish it and never did.

A. HATzES: I remember back in my Doppler imaging days when there were several codes being used to get models of spots, Klaus Strassmeier had a very clever idea. He got all the groups together, he sent them all the same data set, they modelled it not knowing what the other one was getting. He assembled the paper, wrote it and compared the techniques; and it's a highly cited work. So, I would encourage everyone to just do it! Get the groups together, give the people the same data set, and publish a paper making a direct comparison of the result.

R. Wilson: According to my not very specific recollection, there actually have been a number of papers published comparing the light curve models. There typically weren't highly quantitative comments. We ran our program with someone else's program and got basically the same answers. We should do this on a uniform basis including a whole bunch of programs, and not just one or two.

P. EgGleton: I think an early comparison was made by Galileo between the Copernican system and the Ptolemaic system. I think he rather loaded the argument when he called the supporter of Copernicus by the Latin word for 'clever' while the supporter of the Ptolemaic system was the Latin word for 'stupid.' But, there was also an unbiased observer who, being a servant of the Pope, was prepared to accept the stupid ones. My point is, I think it is very important that we all get on with our own work and we should not always be looking over our shoulders at other people, only occasionally. Otherwise, we will be coming to a stop because we will all be so busy looking at everybody else's work. Ultimately, I think the best work will show up by itself.

A. Batten: Since history has been mentioned, I enjoyed Stefan Mochnacki's paper very much because he gave us context and showed us what had to be done without going 
into too much detail. I did not entirely agree, however, that the introduction of lightcurve synthesis was a paradigm shift from the graphical methods of Russell \& Merrill. In between them were the iterative methods developed by Kopal \& Piotrowski. (Incidentally, considering the part of the world in which we are meeting, it is surprising that Kopal has been mentioned only occasionally and Piotrowski not at all until now. They were leaders in the field in their day.) Those methods are not much used today obviously because light curve synthesis is so much better now. Kopal's methods were more used in Europe but never really caught on in North America, perhaps because of the influence of Russell and his intellectual heirs. Possible nationalist considerations sometimes affect the way in which we do science. As Kopal's graduate student, I had no choice but to use his methods in my first paper. However, I would like to draw a parallel between Kopal and Russell. Stefan said that he's beginning to feel like John Merrill. I have often felt that towards the end of his life, Kopal was beginning to feel like Henry Norris Russell. Russell resisted Kopal's iterative methods and was strongly opposed to them. If you have read Kopal's autobiography, you know that story pretty well. Then, towards the end of his life, Kopal equally resisted the light curve synthesis methods. I think he was wrong in doing so, but until the end of his days he wouldn't accept that light curve synthesis was the way to go. And as you all know, he developed his Fourier transform methods, which one or two people have used, but they don't seem to have caught on. I think it's interesting to draw this parallel between Henry Norris Russell on one hand and Kopal on the other hand.

R. WiLson: Well, Kopal had really three ways of supposedly improving on the analysis problem. The first one would be focusing entirely on the eclipses and ignoring the rest of the light curve. They were purely simple geometrical operations with spheres and so on. Then, there were his corrected spheres model and that would've been useful if they had caught on; useful, I would say for about 5 years, 10 at the most. It didn't catch on, so it missed the technological niche. And then, there is the third one that you just mentioned about the Fourier methods. I realized that it was always flying against its limits from the beginning, because if you had too many Fourier terms, you wound up measuring the noise, and if you didn't have enough terms then you were not fitting the function. So, this method was generally not very effective.

E. Budding: Alan, myself, and at least three of us here on this august panel were pupils of the late Zdenek Kopal. One of the things that comes to my mind most strongly about him was his remarkable sense of humor. I recall as a young postgraduate student showing him one of my first light-curve solution efforts, which included things like 'inclination = 80.1234 deg.' After he had a careful look through my sheets, came the response: "Err, Ed, the 4 may well be correct - but the 8 is doubtful."

This morning, we had discussions about the alternative methods of fitting spectra, which I felt was a complimentary and non-adversarial, constructive sort of approach to how different codes can be suited to different contexts and can be complementary. This seems to contrast with the rather gladiatorial or adversarial atmosphere seen in photometric modelling.

Someone cited a quotation at today's meeting, I think from von Neumann, "If you give me four parameters I can model an elephant, and if you give me five parameters I can make him wiggle his tail." It seems that the situation we have is like an elephant seen through a glass darkly. We don't really know if that elephant wants to wiggle its tail. It knows, but we should not say we know what that elephant wants to do. What I am trying to say is that we should not try to get more parameters from a model than the information can really tell you. 
P. HaRmaneC: While I was listening to the talks here for several days, I was wondering: Are we not moving for some time in a kind of vicious circle? I do not doubt that analysis, let's say we have radial velocity curves or light curves which are very accurate, so formally the accuracy of the parameters (masses or radii) which you get from them are really very accurate. But, we also heard about models which were calculated for 1.5 years and yet there is one parameter which relates to the still not ideal theory for the mixing length parameter. I was surprised that the mixing length used for cool stars was set at 1 or 1.25. But, if you look in recent models, if you try to reproduce our Sun, you need to use a mixing length parameter close to 2 . Will you produce also a series of computations for one model again for several months to try also this parameter? Because I'm interested in hot stars, then you have to include rotation, and now the problem of how to compare observations with the data becomes more serious. Also, if you want to compare results with the data, we have to make several steps. For instance, how accurate are the bolometric corrections? Basically, they are sometimes based on emission-lined stars which were measured with an intensity interferometer. So, are you sure that we are not getting the answers which we sometimes put into the data in advance?

I. Hubeny: I don't think we are moving in circles. I think we are really moving forward. For instance, as far as convection is concerned, there was some sort of confusion or misunderstanding because the models like COBOLD or Asplund/Nordlund models reproduce convective motions from first principles. It means that you can translate their results into language using the mixing length parameter. Similarily, some simulations for accretion disks done on the box can reproduce the value of the alpha parameter of Shakura-Sunyaev, which doesn't mean that they use it. The real endeavour is to use physics from first principles to do simulations and to derive previously ad hoc parameters, which are no more $a d$ hoc. So, I think the program is very sound and in this respect I'm almost sure that progress proceeds in a very right way. Now, of course doing hydro is very difficult, therefore something always has to be sacrificed. There is always something which is unknown. So the question is: What are we sacrificing more? Or are we sacrificing something more important than what we want to determine? Only experience will tell. But, as far as model atmospheres for cool stars is concerned, I think we are generally as a community in the right thread, because there was a lot of good progress toward understanding motions and understanding convection. A very similar situation occurred in accretion disk modeling, because there was a long stagnation after the Shakura-Sunyaev alpha parameter was introduced. For three decades, people were using it, knowing that it was sort of an ad hoc parameter, and after three decades finally people did simulations where one can get rid of it. So, I agree with you that part of the simulation is always uncertain because something has to be sacrificed, you cannot model everything, you cannot put in your hydro models everything. But, overall, I think that progress is being made.

S. Rucinski: I would like to continue with something that Petr actually brought up. It is this big discrepancy between observational results; a difference of more than 3 sigma or 14 sigma was mentioned. What I'm concerned about is that in many cases we don't know how to do error analysis correctly. Let's admit this. This sigma is a concept that should slowly be put into the annals as useful concepts, like the names of our distinguished predecessors - on whose shoulders we are sitting. But, we have better tools for doing statistical analysis of data. I'm very much for just improving our error analysis including systematic errors and such things that astronomers produce and can do it so well. Let's look at different concepts like uncertainty levels and so on, instead of sigma. 
W. KLEY: Coming back to the point which was raised before. You answered it a bit already. I think the situation is bad and good on both sides. It's clear that to produce stellar evolution models or stellar atmosphere models you need to have some quantities like the alpha (mixing length) parameter, which summarizes the uncertainties you have for processes which are 3D, MHD etc. People continue to use that and I think this is of course very good. The bad thing is that you don't really know the value of the full parameter. The other thing is that you don't even know whether the parametrization is correct in the first place. I don't mean the parameter itself, but the whole theory on it. It may not be correct. The same thing applies to the alpha parameter in accretion disk theory since people have been using the alpha parameter for a long time. But, all the uncertainties about the turbulence, whether it's driven by the hydrodynamic turbulence or by full MHD turbulence, has been summarized in one constant number. Presently, it's not even clear if this approach is correct at all. This is the downside of these approaches. The positive side of this is that while we have learned a lot, we will be able to clarify the situation with advanced modelling. Then we can say in hindsight basically: "Well, this was a good idea" or "This was a bad idea." There may be corrections, but one has to keep this in mind. There is progress.

V. TRimble: There may be something to be learned from the medical community, who have been working hard on statistics now for a long time, and whose conclusions about whether something is statistically significant are perhaps more important than ours. But, they define their criteria of what will be regarded as agreement or disagreement before they start doing the calculations or collecting the data. And, when they decide that something is significant or is not, then they stop.

A. LinnelL: Back to Slavek's points. I'd like to bring up again the value of numerical experiments. I think that one of the very valuable things that can be done in testing a particular routine is to generate synthetic data, which you then solve. You can modulate the data with assignable errors and then determine whether or not your solution technique can recover the data to within the error that you expect. You can vary all sorts of parameters, but if you construct a model which you test, where you control the parameters, then you're in a position to say whether you can recover them to within the accuracy that you would expect. I think that is a very valuable test.

P. Bonifacio: Unfortunately, in many cases, this underestimates your error. We were always very good at taking our models, adding some noise plus something, and recovering the correct parameters. Except when you get the real data, there's not only Poisson noise, it's more complex. But, I agree that it's a very useful experiment.

P. STEE: Another way to do this is to do it in a 'blind mode.' I mean to distribute the same data to other people. We have done this for example with interferometry. We call it the 'beauty contest.' You can identify the best code if you have data that you don't know and you try to reconstruct the images from the data with the different algorithms.

R. WiLson: If you wanted to know how well the models compare, then you have to run the various models for the identical parameters. If you want to know how much the answers depend on the physical constants that go into the code, then you vary the physical constants and see what you get. If you want to know the overall errors after you know those two questions and several other questions, then there are simple little formulas. The simplest method is to just take the rms result and put the results together, 
and if it's more complicated than that, you may have to take correlations into account but there are simple little statistical formulas for doing that. You have to look at the problems one by one and then combine them at the end.

A. BAtten: I would like to make some comments on Mercedes Richards' paper of yesterday morning. This is beautiful work and fills me with admiration tinged with envy. That was the sort of thing I wanted to do 40 years ago with U Cephei and could not. I also suspected that magnetic forces were involved somehow, as Mercedes has clearly shown. A few of us here were also at the 1975 conference in Cambridge where the criticism was made that none of the trajectories proposed for gas-streams obeyed Newton's laws of motion. I think that implicit in that remark was the assumption that only gravitational forces were acting on the stream. We did not know then, and are only now beginning to find out, how other forces may act. I hope Mercedes will study U Cephei soon, while I am still alive to see the result!

E. Devinney: A few comments: I was almost blown away by the ambition of people, what kind of problems are being attacked these days. It seems like these problems are growing in complexity with many features: disks, clouds, etc., including new physics. I think that makes the idea of testing and verifying codes much more difficult than it has been in the past. I also really enjoyed Jan Budaj's talk particularily, because of the explicit link between the exoplanets and binary paradigms.

V. TRImble: The IAU met in Prague in 1967, a few people who were there are here. But there was the first set of talks about interacting binaries and the evolutionary scenarios that they predicted. From Kippenhahn, from Mirek Plavec and from Bohdan Paczynski, who in the end took the heat when Anne Underhill stood up and said "There are more models that are not stars than there are stars that are not models." This is probably still true.

K. BJorkman: We've heard a lot today about how our models and our observations are both outstripping the fundamental data on which we need to base our interpretation. I'm curious as to your thoughts on whether that message is getting through to the funding agencies. I also worry a lot when I see a lot of our fundamental atomic data specialists retiring and not being replaced. So, I'm wondering where these improved atomic data are going to come from?

E. BudDING: Someone asked me to mention that it is good to have more communications like this where these points can be made. Perhaps through the medium of something like EVO where people with these needs in mind can form a collective voice to emphasize them and do so repeatedly. Such meetings are held for the Australian SKA Pioneer project at least once a month, if not more often. When the requirements are reiterated, people can't avoid finding out about it.

V. TRImble: IAU Working Groups can be assembled for that precise kind of purpose. It requires two or three people who feel that this is important. They could easily in Beijing put forward a proposal for a Working Group that would probably communicate mostly online but would get together when possible.

I. HuBENY: There are conferences in the US organized every two years about laboratory astrophysics and atomic data. This group has some voice but never a real impact. If you sit on an Astrophysics Theory Panel (ATP) for NASA, there are about a hundred 
proposals and only five of them will be funded. So, the probability that even a very good proposal for atomic or molecular data will be funded is very low. When I was on the panel, I tried very hard to push them, but I must say I didn't succeed very often, actually only once. But, it is very hard to get funding because it's not astrophysics. Unfortunately, it is falling between the cracks, even if we need it.

A. Burrows: I was going to say the same thing and add a comment. This comes up all the time. Laboratory astrophysics and doing the fundamental microphysical calculations are central to just about everything we do. But, that effort is not well-credited. There are very few tenured people who know how to do any of this. The chemistry community that might be able to help in some aspects doesn't care about this sort of thing at all. A lot of the calculations that are done, are done in national labs by people on soft money. It's not quite the same in Europe, because Europeans do support this a little bit more, but on both continents it's not supported as much as needed, and I don't think the situation is going to change at all. Voices could be raised, there is an awful lot of data that is necessary, but it's deemed fundamentally as a drudgery by tenure committees and people who are interested in advancing astrophysics. And, it is just going to get worse. I don't want to be too pessimistic, but we've seen this situation evolving over the years. Many people have talked about this, even the decadal surveys have talked about this. They always mention or give lip service to laboratory astrophysics getting microphysical data. Every once in a while there are some advances, for example, opacities for iron. You can add a few things, but just think of how many ionic states, how many molecules, how many line transitions, how many collisional rates for processes, we have to think about. Also, think how difficult it is to do the experiment to just measure one of those. It's going to be difficult.

P. SkODA: There is already a project that is well-funded by the European Union, it's called the Virtual Atomic and Molecular Data Center. It is exactly the attempt to concentrate all the available atomic and molecular databases together and provide a unified interface to access the data. For example, in 2012 there will be a Summer School in Serbia about teaching the students how to operate with such new databases. There are in fact billions of the transitions already available through these systems. So, it's a question of the informatics approach to get all the particle data in the proper way in a machine readable format.

I. Hubeny: My understanding is that they will collect what's already done: the opacity project. However, we need NEW data.

J. HiLliER: One of the things in stellar atmospheres that you can't get from theory are the wavelengths. Theory just cannot predict wavelengths accurately enough. We've talked about the oxygen problem today and it's a nickel line that was one of the contributions to that problem. If we don't know a line is there, you can't correct for one. It's as simple as that. As Ivan mentioned, the High Energy Laboratory Astrophysics Group meets every two years. There are people in that group who are willing to work with you to get the laboratory data if you can demonstrate a need for a particular problem. That is sometimes difficult, so it takes time.

P. Bonifacio: These things are good, but it will not happen unless we make the laboratory astrophysics and theoretical computations attractive for young people to enter in the field; that means you must provide careers, money, and infrastructure. So, we're losing 
expertise. For quantum-chemical computations, one thing I want very desperately are computations for cross-sections with collisions with hydrogen atoms. We have lithium, sodium, now magnesium, and it takes years to get the next element.

I. Hubeny: I agree also with John's comments. I heard that too. However, when people ask me for a model, I say "Sure, I can do that." I can in principle, but it takes me sometimes weeks or months, to be honest. Most people also have their own projects and everything. So, in principle, they are willing, but in reality it all goes very slowly.

A. Batten: The picture of terrestrial "brown dwarfs" shown this morning reminded me of a story Willem Luyten loved to tell. He was a great authority on white dwarfs. For many years, he submitted proposals to the National Science Foundation and received grants for his research on white dwarfs. One day, a new bureaucrat, who knew neither Luyten nor astronomy, sent him a letter asking, "Does this proposal involve research on human subjects?"

V. Trimble: Alan has just said it, but if you knew Willem Luyten, the answer to that to a certain extent was "Yes."

M. ZEJDA: We live in time when we are hunting for better accuracy in measurements of brightnesses, fluxes, and radial velocities. We use new measurements and also archival values to study long-term variations. The time-base for these long-term studies is usually given in Julian Date with the heliocentric correction (HJD) based on the Coordinated Universal Time (UTC). People use data spread over decades to find differences on a scale smaller than one minute. However, they do not take into account that the accuracy of the timeline based on UTC is not the same! There are leap seconds which caused discontinuous differences of tenths of seconds for data spread over intervals longer then several decades. The solution is quite simple. All authors should publish new studies of any astronomical events at least in the fields of variable stars and exoplanets in the Barycentric Julian Date in the Barycentric Dynamical Time (BJDTBC). Referees should insist on using BJDTBC. The Barycentric Julian Date derived from the Barycentric Dynamical Time should become a new standard. All dates should be recalculated into BJDTBC when old data from an archive are used. As an excellent example of correct work with data, see the paper by Potter et al. 2011, MNRAS, 416, 2202. Another hidden problem in using data archives is that the format of time is different in each archive, so users should be very careful when processing archival data. For more details, see the Appendix in Eastman, Siverd \& Gaudi, 2010, PASP 122, 935; or Zejda \& Domingo, 2011, IBVS 5996. 